\title{
Use of somatostatin in the management of pancreatic haemobilia
}

\author{
M.V. Tobin, S.A. Jenkins, J.R. Gosney and Ian T. Gilmore \\ Gastroenterology Unit, Royal Liverpool Hospital, Liverpool L7 8XP, UK.
}

\begin{abstract}
Summary: An elderly man, not previously known to have chronic pancreatitis, presented with haematemesis and melaena which was endoscopically diagnosed as haemobilia. Retrograde cholangiopancreatography showed blood clot in both the common bile duct and the pancreatic duct and the computed tomographic scan appearances were those of gross calcific chronic pancreatitis. Despite active bleeding, it was not possible to demonstrate its source at angiography, thus precluding therapeutic embolization. Thirty six hours after commencing an infusion of somatostatin, repeat endoscopy showed no evidence of active or recent bleeding. The infusion was continued for 5 days during which time he had no further bleeding.
\end{abstract}

\section{Introduction}

Gastrointestinal haemorrhage occurs in approximately $9 \%$ of patients with chronic pancreatitis. ${ }^{1}$ When severe, it is usually secondary to erosion of a visceral vessel, often in association with pseudocyst formation, with haemorrhage occurring into the stomach, ${ }^{2}$ duodenum ${ }^{3}$ or colon. ${ }^{4}$ Less commonly, bleeding occurs directly into the pancreatic duct and presents as pancreatic haemobilia. ${ }^{5}$ We report a case of endoscopically diagnosed haemobilia as the presenting feature of chronic pancreatitis and describe its treatment with somatostatin.

\section{Case report}

An 84 year old previously healthy man presented with a 4-day history of melaena and had one episode of haematemesis on the day of admission to hospital. He had consumed up to 30 units of alcohol daily throughout his working life but had remained virtually abstinent for the past 5 years. He had no symptoms suggestive of chronic pancreatitis and, apart from pallor, physical examination was normal. Haemoglobin on admission was $6.8 \mathrm{~g} / \mathrm{dl}$. At upper gastrointestinal endoscopy the oesophagus, stomach and duodenal cap were normal but fresh blood was oozing from the papilla of Vater. Retrograde cholangiopancreatography demonstrated multiple radiolucent filling defects consistent with clot formation in both

Correspondence: M.V. Tobin, M.B., M.R.C.P., Broadgreen Hospital, Thomas Drive, Liverpool L14 3LB.

Accepted: 19 October 1988 the common bile duct and pancreatic duct. However, neither coeliac nor superior mesenteric arteriography could identify the bleeding point. A subsequent computed tomographic (CT) scan showed gross calcific pancreatitis particularly affecting the head and uncinate process. He was transfused 4 units of blood and remained haemodynamically stable. However, repeat endoscopy on the following day showed persistent haemorrhage. Surgical treatment was thought inappropriate in view of his age and extent of disease, and therapeutic embolization was precluded by the absence of a visible bleeding vessel on angiography.

Because of the splanchnic vasoconstrictive effect of somatostatin in normal subjects ${ }^{6}$ and our own experience of its value in the control of acute variceal haemorrhage, ${ }^{7}$ an infusion was commenced at a rate of $250 \mu \mathrm{g} / \mathrm{h}$ and a bolus dose, also of $250 \mu \mathrm{g}$, was given daily in addition. Endoscopy performed at 12 hours showed persistent, albeit less severe, bleeding but a repeat examination 24 hours later was entirely normal. The infusion was continued for 5 days and he had no further episode of bleeding throughout his hospital stay. He maintained his haemoglobin above $13 \mathrm{~g} / \mathrm{dl}$ after receiving a total of 8 units of blood in the first 72 hours after admission.

Six weeks following discharge, he was readmitted following a further bleed and died suddenly while being resuscitated.

At post-mortem examination the pancreas was expanded by a large haematoma in the body. Other areas of less extensive haemorrhage were also present with the whole gland showing marked calcific deposits and areas of fibrosis consistent with chronic pancreatitis. No pseudocyst was demonstrated and the 
source of haemorrhage was traced to an erosion of the splenic artery in the middle third of the pancreas. No other abnormality was discovered at histological examination.

\section{Discussion}

Bleeding into the biliary tree was first reported over 300 years ago but only in 1948 did Sandblom introduce the term haemobilia. ${ }^{8}$ Blood entering the biliary tree from the pancreas accounts for only $2 \%$ of cases 9 and is usually due to erosion of a visceral vessel, frequently in association with pseudocyst formation, ${ }^{2,3}$ or aneurysmal rupture. ${ }^{10}$ Diagnosis by direct observation at endoscopy is unusual but has been reported when upper gastrointestinal haemorrhage has occured in patients with known chronic pancreatitis., 5 Coeliac axis or superior mesenteric angiography is

\section{References}

1. Howat, H.J. \& Sarles, H. The Exocrine Pancreas. W.B. Saunders, Philadelphia, 1979.

2. Schecter, L.M., Gordon, H.E. \& Passaro, E. Massive haemorrhage from the coeliac axis in pancreatitis. Am J Surg 1974, 128: 301-305.

3. Gadacz, T.R., Trunkey, D. \& Kieffer, R.F. Visceral vessel erosion associated with pancreatitis. Arch Surg 1978, 113: $1438-1440$.

4. Breitenecker, R. Fatal gastrointestinal haemorrhage due to chronic relapsing pancreatitis. N Engl J Med 1959, 260: $1167-1169$.

5. Brintall, B.B., Laidlaw, W.W. \& Papp, J.P. Hemobilia: pancreatic pseudocyst hemorrhage demonstrated by endoscopy and arteriography. Am J Dig Dis 1974, 19: 186- 188 .

6. Sonnenberg, G.E., Keller, U., Perruchoud, A. et al. Effect of somatostatin on splanchnic hemodynamics in patients with cirrhosis of the liver and in normal subjects. Gastroenterology 1981, 80: 526-532.

7. Jenkins, S.A., Baxter, J.N., Corbett, W. et al. A prospectively randomised controlled clinical trial comparing somatostatin and vasopressin in controlling acute variceal haemorrhage. $\mathrm{Br}$ Med J 1985, 290: 275-278.

8. Sandblom, P. Hemorrhage into the biliary tract following trauma - 'traumatic hemobilia'. Surgery 1948, 24: $571-586$. often successful in locating the source of bleeding, with the splenic artery the most frequently involved vesse ${ }^{3}$ In the absence of an angiographically visible bleedir point which is amenable to embolization, surgical ligation or pancreatic resection is necessary.

Somatostatin, a tetradecapeptide abundantly pre $\overrightarrow{\vec{s}}$ ent in the gastrointestinal tract and pancreas, is $\frac{\text { F }}{7}$ potent inhibitor of both basal and stimulated exocrime pancreatic secretion ${ }^{12,13}$ and reduces pancreatic bloo flow by approximately $30 \%{ }^{6,14}$ Furthermore, it h been shown to be of benefit in the treatment \&f haemorrhagic pancreatitis in the dog. ${ }^{15}$ It therefore seemed reasonable to use somatostatin in an attem $\overrightarrow{0}$ to control the bleeding in this patient whose age precluded extensive surgical resection. The favourable outcome, albeit temporary, suggests that somatostat may be of some value in selected cases of pancreat haemorrhage where embolization is not feasible and surgery potentially hazardous.

9. Sandblom, P. Haemobilia: History. Pathology. Diag nosis. Treatment. Charles C. Thomas, Springfield Illinois, 1972.

10. Bowers, J., Koehler, P.R., Hammar, S.P. et al. Rupture of a splenic artery aneurysm into the pancreatic duab Gastroenterology 1976, 70: 1152-1155.

11. Rosch, W. \& Schaffner, O. Massive gastrointestina haemorrhage into the pancreatic duct - diagnosiके duodenoscopy and ERCP. Endoscopy 1976, 8: 93 65

12. Raptis, S., Schlegel, W., Lehmann, E. et al. Effects of somatostatin on the exocrine pancreas and the release duodenal hormones. Metabolism 1978, 27 (suppl $1321-1328$

13. Dollinger, H.C., Raptis, S. \& Pfeiffer, E.F. Effects $\overline{\vec{F}}$ somatostatin on exocrine and endocrine pancreatic function stimulated by intestinal hormones in man. Hor Metab Res 1975, 8: 74-78.

14. Keller, U., Sonnenberg, G.E., Kayasseh, L. et at Dosisabhangigkeit der Wirkung von somatostatin a die splanchnische Durchblutung beim Mensche Schweiz Med Wochenschr 1979, 109: 595-596.

15. Schwedes, U., Althoff, P.H., Klempa, L. et al. Effects of somatostatin on bile induced haemorrhagic pancreatitis in the dog. Horm Metab Res 1979, 11: 647. 